\title{
Commissioning of Underground Nuclear Astrophysics Ex- periment JUNA in China
}

Weiping Liu ${ }^{1, *}$, Zhihong $\mathrm{Li}^{1}$, Jianjun $\mathrm{He}^{2}$, Xiaodong Tang ${ }^{3}$, Gang Lian ${ }^{1}$, Jun $\mathrm{Su}^{2}$, Yangping Shen ${ }^{1}$, Zhu $\mathrm{An}^{4}$, Fuqiang Chao ${ }^{1}$, Jianjun Chang ${ }^{3}$, Lihua Chen ${ }^{1}$, Han Chen $^{3}$, Xiongjun Chen ${ }^{1}$, Yunhua Chen ${ }^{5}$, Zhijun $\mathrm{Chen}^{3}$, Jianping Cheng ${ }^{2}$, Baoqun $\mathrm{Cui}^{1}$, Xiao Fang ${ }^{6}$, Changbo $\mathrm{Fu}^{7}$, Lin $\mathrm{Gan}^{8}$, Bing Guo ${ }^{1}$, Zhiyu Han ${ }^{1}$, Xuyuan Guo ${ }^{5}$, Guozhu $\mathrm{He}^{1}$, Jinrong $\mathrm{He}^{5}$, Alexander Heger ${ }^{9}$, Suqing Hou $^{3}$, Hanxiong Huang ${ }^{3}$, Ning Huang ${ }^{4}$, Baolu $\mathrm{Jia}^{3}$, Liyang Jiang ${ }^{1}$, Shigeru Kubono ${ }^{10}$, Jianmin $\mathrm{Li}^{11}$, Mingchuan $\mathrm{Li}^{5}$, Kuoang $\mathrm{Li}^{1}$, Ertao Li ${ }^{8}$, Tao $\mathrm{Li}^{3}$, Yunju $\mathrm{Li}^{1}$, Maria Lugaro ${ }^{12}, X i$ aobing $\mathrm{Luo}^{4}$, Hongyi $\mathrm{Ma}^{3}$, Shaobo $\mathrm{Ma}^{3}$, Dongming Mei ${ }^{13}$, Wei $\mathrm{Nan}^{1}$, Weike $\mathrm{Nan}^{1}$, Ningchun $\mathrm{Qi}^{5}$, Yongzhong Qian ${ }^{14}$, Jiuchang Qin ${ }^{1}$, Jie Ren $^{1}$, Changsong Shang ${ }^{5}$, Liangting Sun ${ }^{3}$, Wenliang Sun ${ }^{5}$, Wanpeng Tan ${ }^{15}$, Isao Tanihata ${ }^{16}$, Shuo Wang ${ }^{17}$, Peng Wang ${ }^{4}$, Youbao Wang ${ }^{1}$, Qi $\mathrm{Wu}^{3}$, Shiwei $\mathrm{Xu}^{3}$, Yao Yang ${ }^{3}$, Xiangqing $\mathrm{Yu}^{3}$, Qian Yue ${ }^{11}$, Sheng Zeng ${ }^{1}$, Long Zhang ${ }^{1}$, Hao Zhang $^{1}$, Huanyu Zhang ${ }^{1}$, Liyong Zhang ${ }^{2}$, Ningtao Zhang ${ }^{3}$, Peng Zhang ${ }^{5}$, Qiwei Zhang ${ }^{1}$, Tao Zhang $^{7}$, Xiaopeng Zhang ${ }^{18}$, Xuezhen Zhang ${ }^{3}$, Wei Zhao ${ }^{11}$, Jifang Zhou ${ }^{5}$, and Yong Zhou ${ }^{5}$

${ }^{1}$ China Institute of Atomic Energy, Beijing, China

${ }^{2}$ Beijing Normal University, Beijing, China

${ }^{3}$ Institute of Modern Physics, Lanzhou, China

${ }^{4}$ Sichuan University, Chengdu, China

${ }^{5}$ Yalong River Hydro-Electronic Power Limited, Sichuan, China

${ }^{6}$ Zhongshan University, Guangzhou, China

${ }^{7}$ Shanghai Jiaotong University, Shanghai, China

${ }^{8}$ Shenzhen University, Shenzhen, China

${ }^{9}$ Monash University, Melbourne, Victoria, Australia

${ }^{10}$ RIKEN, Institute of Physical and Chemical Research, Wako, Japan

${ }^{11}$ Tsinghua University, Beijing, China

${ }^{12}$ Konkoly Observatory of the Hungarian Academy of sciences, Hungary

${ }^{13}$ South Dakota State University, Brookings, South Dakota, US

${ }^{14}$ Minnesota University, Minneapolis and Saint Paul, Minnesota, US

${ }^{15}$ University of Notre Dame, Notre Dame, Indiana, US

${ }^{16}$ Osaka University, Suita, Osaka, Japan

${ }^{17}$ Shandong University, Weihai campus, China

${ }^{18}$ Institute for High Energy Physics, Beijing, China

\begin{abstract}
Underground Nuclear Astrophysics Experiment in China (JUNA) has been commissioned by taking the advantage of the ultra-low background in Jinping underground lab. High current $\mathrm{mA}$ level $400 \mathrm{KV}$ accelerator with an ECR source and BGO detectors were commissioned. JUNA studies directly a number of nuclear reactions important to hydrostatic stellar evolution at their relevant stellar energies. In the first quarter of 2021, JUNA performed the direct measurements of ${ }^{25} \mathrm{Mg}(\mathrm{p}, \gamma){ }^{26} \mathrm{Al},{ }^{19} \mathrm{~F}(\mathrm{p}, \alpha){ }^{16} \mathrm{O},{ }^{13} \mathrm{C}(\alpha, \mathrm{n}){ }^{16} \mathrm{O}$ and ${ }^{12} \mathrm{C}(\alpha, \gamma){ }^{16} \mathrm{O}$ near the Gamow window. The experimental results reflect the potential of JUNA
\end{abstract}

*e-mail: wpliu@ciae.ac.cn 
with higher statistics, precision and sensitivity of the data. The preliminary results of JUNA experiment and future plan are given.

\section{Underground physics}

Direct measurement of the cross sections for the key nuclear reactions crucial to hydrostatic stellar evolution within the Gamow window is important for obtaining benchmark data for stellar calculation, verifying extrapolation method, constraining theoretical calculations, explaining the abundance observations and solving key scientific questions in nuclear astrophysics[1].

The direct measurement of astrophysical reaction rates on stable nuclei that require highintensity beams and extremely low background represents a major challenge at the frontiers of nuclear astrophysics due to the cosmic ray background and extremely low cross section, thus the combination of underground laboratory and high exposure accelerator/detector complex is the only solution. The first underground based low-energy accelerator facility, LUNA [2,3] at Gran Sasso underground laboratory has successfully demonstrated the feasibility of meeting these challenges. Encouraged by the LUNA success, underground nuclear astrophysics has become one of the frontiers in the field of nuclear astrophysics. Relevant research programs are proposed in the long range plan in China, US and Europe, with high priorities, such as LUNA and CASPER.

China Jinping Underground Laboratory (CJPL) was established on the site of a hydropower plants in the Jinping mountain, Sichuan, China[4, 5]. The facility is located near the middle of a traffic tunnel. The facility is shielded by $2400 \mathrm{~m}$ of mainly marble overburden, with radioactively quiet rock. Its ultra-low cosmic ray background, which is about 2 orders of magnitude lower than that in Gran Sasso, makes it into an ideal environment for low background experiments. CJPL phase I (CJPL-I) is now housing CDEX [6] and PandaX dark matter experiments.

CJPL phase II[7] (CJPL-II) is the expansion followed by the success of CJPL-I, it currently available for temporary usage in year 2020-2021. It has much larger scale underground experiments space $\left(300,000 \mathrm{~m}^{3}\right.$ volume), planned to house CDEX-II, PandaX-II, and JUNA[8]. The layout of JUNA in CJPL-II is shown in figure 1. The complete commissioning of CJPL-II is scheduled in March 2023, when all the three experiments can be restarted in a well equipped condition. In December of 2020, the JUNA collaboration installed the accelerator in CJPL before the long-period construction. Four reactions had been studied in the first quarter of 2021. Some preliminary results are presented in the following section.

The JUNA project was initiated in the year of 2015. The first ion source beam of $12 \mathrm{~mA}$ proton was delivered in 2016, and the first accelerated $260 \mathrm{keV} 3 \mathrm{~mA}$ proton beam was delivered in 2017. All the beams reached their design goal in ground lab in 2020, with the intensity of $12 \mathrm{~mA}$ and $2.5 \mathrm{emA}$ for proton and alpha beams in $350 \mathrm{keV}$. In the mean time, HPGe and BGO as well as neutron detectors and high power targets are all ready. With those ready parts we performed a couple test and calibrating experiment for the 4 reactions mentioned above. The test experiments results the energy and efficiency calibration, understand active background and deduce the high energy data for ${ }^{25} \mathrm{Mg}(\mathrm{p}, \gamma){ }^{26} \mathrm{Al}$ and ${ }^{19} \mathrm{~F}\left(\mathrm{p}, \alpha_{\gamma}\right){ }^{16} \mathrm{O}$ reactions. The first underground JUNA beam in CJPL-II A1 hall was delivered in Dec. 2020, with proton beam of $2 \mathrm{~mA}$ and $300 \mathrm{keV}$ from then one JUNA is ready for underground experiments. 


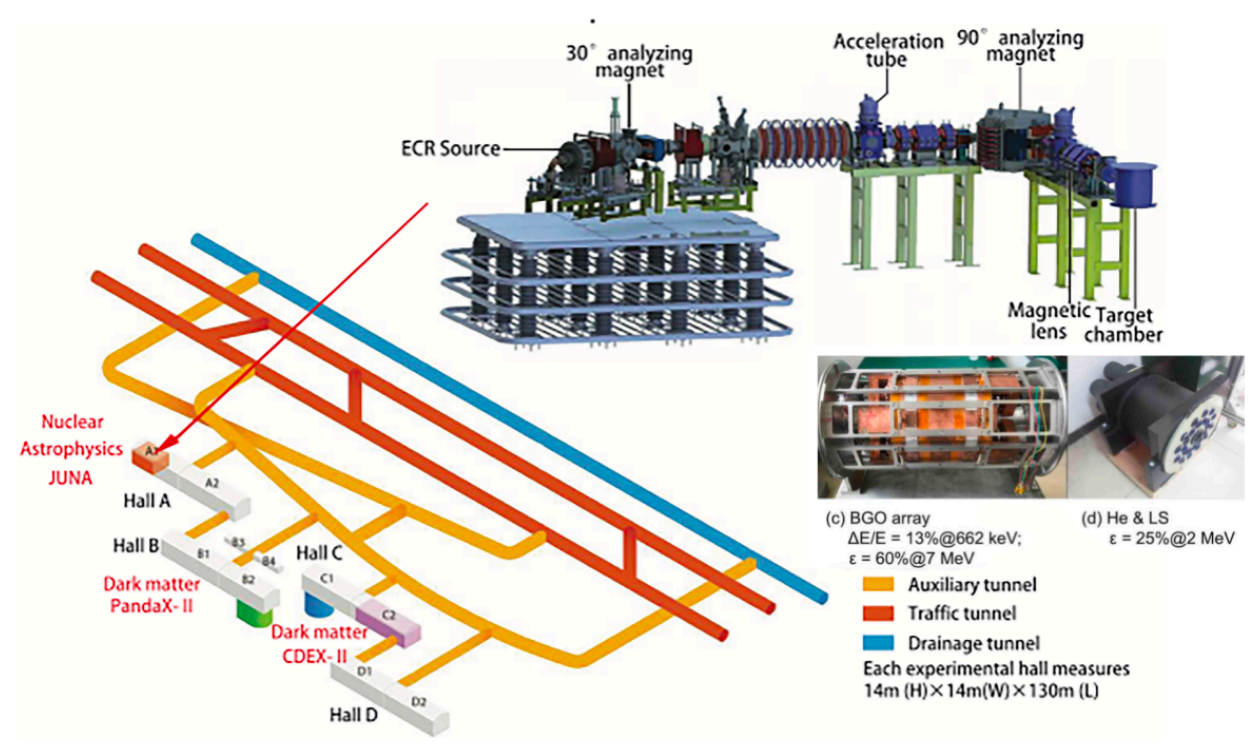

Figure 1. The layout of JUNA in CJPL-II

\section{Nuclear astrophysics reactions measured}

\section{$2.1{ }^{12} \mathbf{C}(\alpha, \gamma){ }^{16} \mathrm{O}$ reaction}

The ${ }^{12} \mathrm{C}(\alpha, \gamma){ }^{16} \mathrm{O}$ reaction is quoted as the holy grail reaction in nuclear astrophysics. The uncertainty of this reaction affects not only the nucleosynthesis of elements up to iron, but also the evolution of the massive stars and their final fate (black hole, neutron star). The cross section of this reaction needs to be determined with an uncertainty of less than $10 \%$ at helium burning temperatures $\left(\mathrm{T}_{9}=0.2\right)$, corresponding to the Gamow window around $\mathrm{E}_{c . m .}=300 \mathrm{keV}$. It is extremely difficult to determine the reaction cross section (about $10^{-17}$ barn) at such low energy. A direct measurement near the Gamow window is performed in JUNA with high intensity ${ }^{4} \mathrm{He}^{2+}$ ion beam to provide better constraints for extrapolating models.

We developed the target with the condition of isotope pure ${ }^{12} \mathrm{C}$ implantation to reduce the disturbance of ${ }^{13} \mathrm{C}$ with the ratio of less than $10^{-5}$. The BGO and $\mathrm{LaBr}$ detector array placed around the target can significantly increase the detection efficiency (absolute efficiency $30 \%$ at $\mathrm{E}_{\gamma}=8 \mathrm{MeV}$ for BGO with fired number selected) of $\gamma$-rays, with the flexibility of anti-coincidence to decrease the beam induced background. We used ${ }^{4} \mathrm{He}^{2+}$ beam with an intensity near $1 \mathrm{emA}$ to achieve the most sensitive upper limit of $10^{-13}$ barn at $\mathrm{E}_{\text {c.m. }}=538$ $\mathrm{keV}$ with potential signal higher than background. The data analysis is still in progress with expert evaluation to pin down the complicated background for either deducing upper limit or significant data points of this reaction near Gamow window, since the accelerator and target induced background is higher than we expected. In the near future we plan to measure this reaction with higher intensity beam, higher purity target and higher vacuum, planned in the coming second stage JUNA run from 2023.

\section{$2.2{ }^{13} \mathrm{C}(\alpha, \mathbf{n}){ }^{16} \mathrm{O}$}

The ${ }^{13} \mathrm{C}(\alpha, n){ }^{16} \mathrm{O}$ reaction is the main neutron source for the slow-neutron-capture (s-) and inter-mediate (i-) processes in AGB stars. Direct measurements at astrophysical energies 
in above-ground laboratories are hindered by the extremely small cross sections and vast cosmic-ray induced background. We performed a direct measurement at JUNA in CJPL using the most intense $\alpha$ beam available among the underground laboratories. The systematic uncertainties of target thickness and deterioration were eliminated using the thick target technique. Our measurement extended from the lowest energy ever measured $\mathrm{E}_{c . m} .=0.24 \mathrm{MeV}$ up to $0.59 \mathrm{MeV}$, covering almost the entire i-process Gamow window with the highest precision. We also performed a consistent R-matrix analysis, which provides a reliable reaction rate for s-process nucleosynthesis with an improved uncertainty[9], see figure $2 b$.
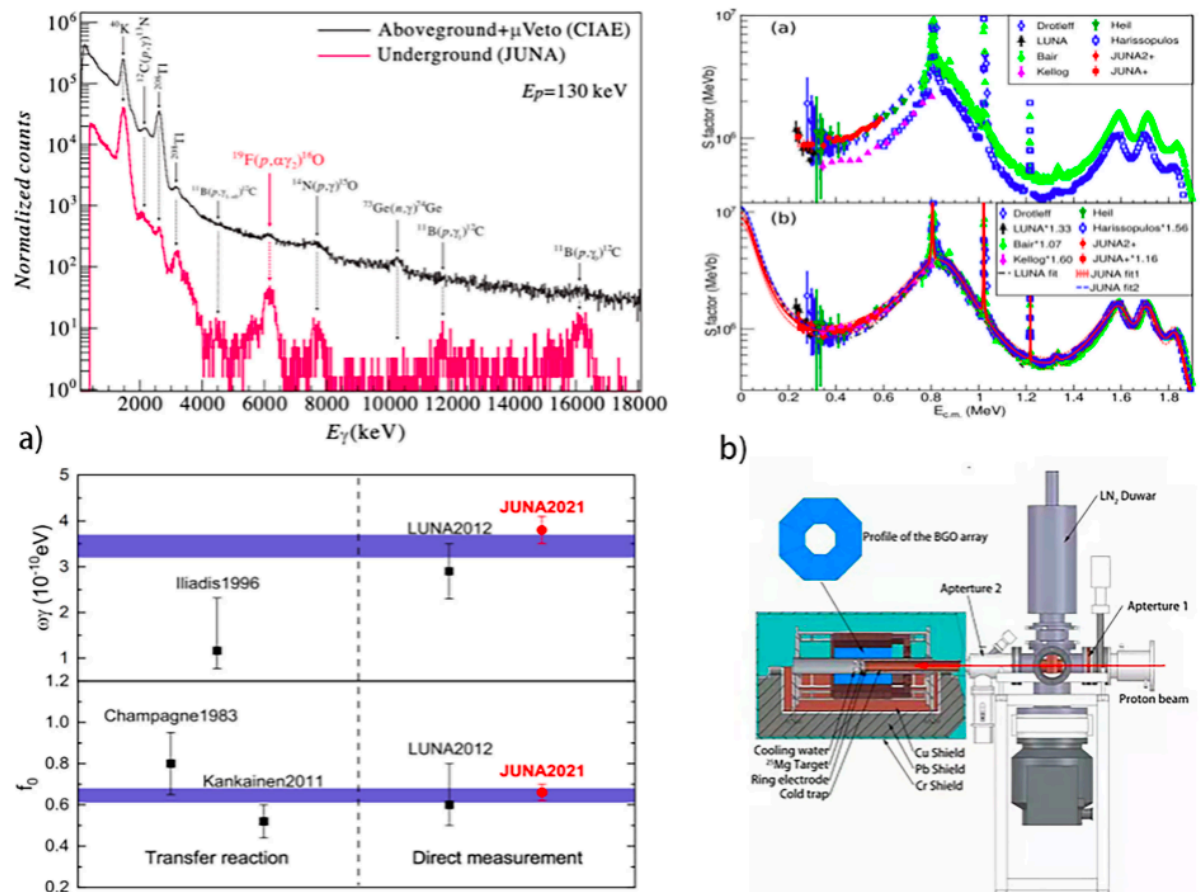

b)

c)

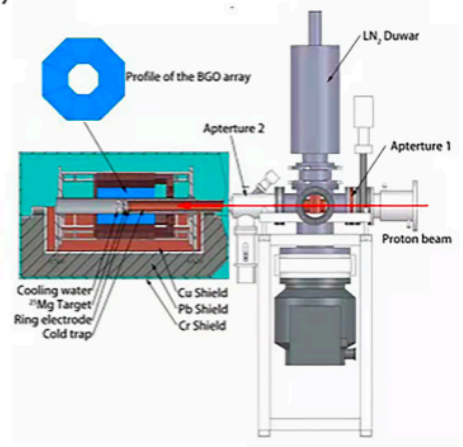

d)

Figure 2. The experiment setup and data analysis

a) $\gamma$-ray spectra of the ${ }^{19} \mathrm{~F}+\mathrm{p}$ experiments measured by a $4 \pi \mathrm{BGO}$ array at a proton energy of $\mathrm{E}_{p}=130 \mathrm{keV}$.

b) The $\mathrm{S}$ factor of the ${ }^{13} \mathrm{C}(\alpha, \mathrm{n}){ }^{16} \mathrm{O}$ reaction (a) without and (b) with normalization factors.

c) Comparison of recent values of the resonance strength and ground state feeding factor of the $92 \mathrm{keV}$ resonance.

d) Schematic diagram of detector setup.

\section{$2.3{ }^{25} \mathrm{Mg}(\mathrm{p}, \gamma)^{26} \mathrm{Al}$}

The ${ }^{25} \mathrm{Mg}(\mathrm{p}, \gamma)^{26} \mathrm{Al}$ reaction plays an important role in the study of cosmic $1.809 \mathrm{MeV} \gamma$ ray as a signature of ongoing nucleosynthesis in the Galaxy. At astrophysical temperature around $0.1 \mathrm{GK}$, the ${ }^{25} \mathrm{Mg}(\mathrm{p}, \gamma)^{26} \mathrm{Al}$ reaction rates are dominated by the $92 \mathrm{keV}$ resonance capture process. We report a precise measurement of the $92 \mathrm{keV}{ }^{25} \mathrm{Mg}(\mathrm{p}, \gamma)^{26} \mathrm{Al}$ resonance in the dayone experiment at JUNA facility in CJPL. The resonance strength and ground state feeding factor are determined to be $(3.8 \pm 0.3) \times 10^{-10} \mathrm{eV}$ and $0.66 \pm 0.04$, see figure $2 \mathrm{c}$, respectively[10]. The results are in agreement with those reported in the previous direct 
underground measurement within uncertainty, but with significantly reduced uncertainties. Consequently, we recommend new ${ }^{25} \mathrm{Mg}(\mathrm{p}, \gamma)^{26} \mathrm{Al}$ reaction rates which are by a factor of 2.4 larger than those adopted in REACLIB database at the temperature around $0.1 \mathrm{GK}$. The new results indicate higher production rates of ${ }^{26 g} \mathrm{Al}$ and the cosmic $1.809 \mathrm{MeV} \gamma$-rays. The implication of the new rates for the understanding of other astrophysical situations is very significant.

The thick-target yields of the 92 and $189 \mathrm{keV}$ resonances of ${ }^{25} \mathrm{Mg}(\mathrm{p}, \gamma){ }^{26} \mathrm{Al}$ were measured with $2 \mathrm{pmA}$ proton beams and $4 \pi$ BGO $\gamma$-ray detector. The event rate of the ${ }^{25} \mathrm{Mg}(\mathrm{p}$, $\gamma)^{26} \mathrm{Al}$ reaction at $\mathrm{E}_{c . m .}=92 \mathrm{keV}$ is about 200 events per day. The background counting rate is measured to be less than 5 events per day. Thus we accumulated around 1200 reaction events in two weeks, and the statistic uncertainty is about $3 \%$ with total exposure of $1225 \mathrm{C}$.

The resonance strength of $58 \mathrm{keV}$ level is estimated by using the shell model calculation and the in-direct measurement by ${ }^{25} \mathrm{Mg}\left({ }^{7} \mathrm{Li},{ }^{6} \mathrm{He}\right){ }^{26} \mathrm{Al}$ one nucleon transfer reaction[11]. The results show that the $58 \mathrm{keV}$ resonance dominate the ${ }^{25} \mathrm{Mg}(\mathrm{p}, \gamma)^{26} \mathrm{Al}$ reaction rate at $\mathrm{T}<0.06$ GK[11]. Thanks for our indirect deduction of $58 \mathrm{keV}$, and our ground based experimental results for $304 \mathrm{keV}$, based on our underground data for 92 and $189 \mathrm{keV}$ resonances, we are able to build up most precise reaction rate for all temperatures, together with that of LUNA data, the results will be published elsewhere.

\section{$2.4{ }^{19} \mathrm{~F}(\mathrm{p}, \alpha){ }^{16} \mathrm{O}$ reaction}

Fluorine is one of the most interesting elements in nuclear astrophysics, where the ${ }^{19} \mathrm{~F}\left(\mathrm{p}, \alpha_{\gamma}\right){ }^{16} \mathrm{O}$ reaction is of crucial importance for Galactic ${ }^{19} \mathrm{~F}$ abundances and CNO cycle loss in first generation Population III stars. As a dayone campaign at the JUNA, we finished direct measurements of the essential ${ }^{19} \mathrm{~F}\left(\mathrm{p}, \alpha_{\gamma}\right){ }^{16} \mathrm{O}$ reaction channel. The $\gamma$-ray yields were measured over $\mathrm{E}_{c . m .}=72.4-344 \mathrm{keV}$, see figure 2a [12], covering the Gamow window; our energy of $72.4 \mathrm{keV}$ is unprecedentedly low, reported here for the first time. The experiment was performed under the extremely low cosmic-ray induced background environment of CJPL, one of the deepest underground laboratories in the world. The present low-energy $\mathrm{S}$ factors deviate significantly from previous theoretical predictions, and the uncertainties are significantly reduced. The thermonuclear ${ }^{19} \mathrm{~F}\left(\mathrm{p}, \alpha_{\gamma}\right){ }^{16} \mathrm{O}$ reaction rate has been determined directly at the relevant astrophysical energies.

Table 1. Basic parameters of four reactions studied

\begin{tabular}{cccccccc}
\hline reaction & beam & $\begin{array}{c}\text { intensity } \\
(\mathrm{emA})\end{array}$ & $\begin{array}{c}\mathrm{E}_{c . m .} \\
(\mathrm{keV})\end{array}$ & cross section & $\begin{array}{c}\text { efficiency } \\
\%\end{array}$ & $\begin{array}{c}\mathrm{CTS} \\
\text { (/day) }\end{array}$ & $\begin{array}{c}\text { BKD } \\
\text { (/day })\end{array}$ \\
\hline${ }^{12} \mathrm{C}(\alpha, \gamma){ }^{16} \mathrm{O}$ & ${ }^{4} \mathrm{He}^{2+}$ & 1 & 538 & $10^{-13}$ barn & 60 & - & 1.2 \\
${ }^{13} \mathrm{C}(\alpha, \mathrm{n}){ }^{16} \mathrm{O}$ & ${ }^{4} \mathrm{He}^{1+, 2+}$ & 2 & $230-600$ & $10^{-12}$ barn & 26 & $97 \pm 24$ & $113 \pm 5$ \\
${ }^{25} \mathrm{Mg}(\mathrm{p}, \gamma){ }^{26} \mathrm{Al}$ & ${ }^{1} \mathrm{H}^{1+}$ & 2 & $\mathrm{E}_{x}=92$ & $\begin{array}{c}(3.8 \pm 0.3) \\
\times 10^{-10} \mathrm{eV}\end{array}$ & 38 & 200 & 10 \\
${ }^{19} \mathrm{~F}\left(\mathrm{p}, \alpha_{\gamma}\right){ }^{16} \mathrm{O}$ & ${ }^{1} \mathrm{H}^{1+}$ & $0.5-1.0$ & $72-344$ & $10^{-12}$ barn & 60 & 105 & 85 \\
\hline
\end{tabular}

\section{Summary}

The parameters of four reactions are summarized in table 1. In summary, a new underground nuclear astrophysics experiment JUNA planned for the expanded space CJPL-II was performed. With a more powerful accelerator and a deeper location, JUNA has joined the research among underground nuclear astrophysics laboratories. The accelerator system and 
detector array was installed by the end of 2020, the experiments started in the beginning 2021 and the first four experimental data accumulated in the first quarter of 2021. The astrophysical implications base on those data is going on, with the published result hopefully available in the middle and end of 2021.

In the coming years before the re-opening in 2023, we will put more efforts to enhance beam intensity, target purity, as well as improving detectors. Future upgrading is planned (JUNA-II), by considering to build a MV level Van de Graaff for ${ }^{12} \mathrm{C}$ and ${ }^{16} \mathrm{O}$ beam in inverse kinematics and window-less targets as well as recoil spectrometers. We welcome the researchers world wide to join JUNA to perform more experiments by using the most favorable underground condition in Jinping.

\section{References}

[1] C. Iliadis, Nuclear Physics of Stars, Wiley-VCH Verlag GmbH (2007)

[2] A. Formicola, G. Imbriani, M. Junker, et al., Nuclear Instruments \& Methods in Physics Research, 507, 609-616 (2003)

[3] H. Costantini, A. Formicola, G. Imbriani, et al., Reports on Progress in Physics, 972, 086301 (2009)

[4] H. S. Chen, Science(in Chinese), 62, 4 (2010)

[5] J. P. Cheng, S. Y. Wu, Q. Yue, et al., Physics (in Chinese), 03, 149-154 (2011)

[6] W. Zhao, Q. Yue, K. J. Kang, et al., Physical Review D, 88, 1201-1205 (2013)

[7] D. Normile, Science, 346, 1041-1041 (2014)Chicago Press (1988)

[8] W. P. Liu, et al., Science China Physics, Mechanics \& Astronomy, 59, 642001-1-6400017(2016)

[9] B. Gao,..., X. D. Tang,..., W. P. Liu, Direct measurement of the ${ }^{13} \mathrm{C}(\alpha, \mathrm{n}){ }^{16} \mathrm{O}$ reaction in the Gamow window of the i-process nucleosynthesis using intense beam in deep underground laboratory, submitted to Phys. Rev. Lett., 2021

[10] J. Su, H. Zhang, Z. H. Li,..., W. P. Liu, First result from the Jinping underground nuclear astrophysics experiment JUNA: precise measurement of the $92 \mathrm{keV}^{25} \mathrm{Mg}(\mathrm{p}, \gamma)^{26} \mathrm{Al}$ resonance, Science Bulletin, 67, 2 (2022)

[11] Z. H. Li, J. Su, Y. J. Li, et al., Science China Physics, Mechanics \& Astronomy, 58 (2015)

[12] L.Y. Zhang, J. Su, J.J. He,..., W. P. Liu, Direct Measurement of the Astrophysical ${ }^{19} \mathrm{~F}\left(\mathrm{p}, \alpha_{\gamma}\right){ }^{16} \mathrm{O}$ Reaction in the Deepest Operational Underground Laboratory, Phys. Rev. Lett. 127, 152702 (2021). 